\title{
Nusinersen treatment in adult patients with spinal muscular atrophy: a safety analysis of laboratory parameters
}

\author{
Benjamin Stolte ${ }^{1,5} \cdot$ Michael Nonnemacher ${ }^{2} \oplus \cdot$ Kathrin Kizina $^{1,5} \cdot$ Saskia Bolz ${ }^{1,5} \cdot$ Andreas Totzeck $^{1,5}$. \\ Andreas Thimm $^{1,5} \cdot$ Bernd Wagner $^{3}$ (1) $\cdot$ Cornelius Deuschl ${ }^{4,5} \cdot$ Christoph Kleinschnitz $^{1,5} \cdot$ Tim Hagenacker $^{1,5}$
}

Received: 2 March 2021 / Revised: 15 April 2021 / Accepted: 16 April 2021 / Published online: 25 April 2021

(c) The Author(s) 2021

\begin{abstract}
Background Nusinersen is an intrathecally administered antisense oligonucleotide (ASO) that improves motor function in patients with spinal muscular atrophy (SMA). In addition to efficacy, the safety of a therapy is the decisive factor for the success of the treatment. For some ASOs, various organ toxicities have been described, such as thrombocytopenia, renal and liver impairment, or coagulation abnormalities. However, systematic data on laboratory parameters under treatment with nusinersen are mainly available from studies in infants and children. Therefore, our aim was to assess the safety of nusinersen therapy in adult SMA patients.

Methods Laboratory data from 404 nusinersen injections performed in 50 adult patients with SMA type 2 and type 3 were retrospectively analyzed.

Results The total observation period was 76.9 patient-years, and patients received up to 12 injections. Our data provides no new safety concerns. In cerebrospinal fluid (CSF), the mean white blood cell count and lactate remained stable over time. Total CSF protein increased by $2.9 \mathrm{mg} / \mathrm{dL}$. No change in mean platelet count was observed under therapy. Only one patient showed sporadic mild thrombocytopenia. Coagulation parameters and inflammatory markers were stable. The mean creatinine level decreased by $0.09 \mathrm{mg} / \mathrm{dL}$. Analysis of mean liver enzyme levels revealed no relevant changes during treatment. Conclusion Our data demonstrate a favorable safety profile of nusinersen therapy in adult SMA patients under longer-term "real-world" conditions. In particular, we found no evidence of clinically relevant platelet declines, coagulopathies, or renal or hepatic organ toxicities, which are common concerns with the use of ASOs.
\end{abstract}

Keywords ASO $\cdot$ Platelets $\cdot$ Kidney $\cdot$ Liver $\cdot$ Coagulation $\cdot$ Toxicity $\cdot$ Side effects

Tim Hagenacker

tim.hagenacker@uk-essen.de

1 Department of Neurology, University Hospital Essen, Hufelandstr. 55, 45147 Essen, Germany

2 Institute for Medical Informatics, Biometrics and Epidemiology, University Hospital Essen, Essen, Germany

3 Department of Clinical Chemistry, University Hospital Essen, Essen, Germany

4 Institute for Diagnostic and Interventional Radiology and Neuroradiology, University Hospital Essen, Essen, Germany

5 Center for Translational and Behavioral Neuroscience, University Hospital Essen, Essen, Germany

\section{Introduction}

The condition, $5 \mathrm{q}$-associated spinal muscular atrophy (5qSMA) is a rare genetic neuromuscular disorder that leads to progressive muscle atrophy and weakness because of degeneration of anterior horn cells [28]. The "classic" clinical classification of the disease is based on the achievement of motor milestones during childhood, with a broad range of phenotypes from severely affected infants never achieving the ability to sit (SMA type 1), to individuals able to sit but never achieving the ability to walk (SMA type 2), to ambulatory patients (SMA type 3) [31].

The disorder 5q-SMA is caused by a homozygous deletion or mutation in the survival of the motor neuron 1 gene (SMN1) localized on chromosome $5 \mathrm{q}$, which results in insufficient levels of SMN proteins[24, 25]. Nusinersen is an 
antisense oligonucleotide (ASO) that is capable of increasing SMN protein production.

In clinical trials, intrathecal treatment with nusinersen led to meaningful benefits on motor functions in infants and children[14, 29]. Based on the results of these studies, nusinersen was approved by the US Food and Drug Administration (FDA) and the European Medicines Agency (EMA). Moreover, both the feasibility and efficacy of nusinersen therapy have also been well established in adult SMA patients [18, 26, 37, 39, 42, 43].

In addition to feasibility and efficacy, the safety of a therapy is the decisive factor for the success of the treatment. In the past, various organ toxicities have been described with the use of some ASOs, such as an increase in liver transaminases, renal impairment, coagulation abnormalities, or thrombocytopenia $[3,7,15,20]$. Since ASOs are not able to cross the blood-brain barrier (BBB), nusinersen is administered intrathecally [16]. In terms of potential side effects, this factor could make a difference compared to other ASOs.

To date, systematic data on laboratory parameters under treatment with nusinersen are still scarce and are mainly available from pivotal studies in infants and children [11, 38]. Here, we present our data on laboratory findings in adult SMA patients under treatment with nusinersen. These data are valuable to better assess the safety of longer-term nusinersen therapy in this patient cohort.

\section{Materials and methods}

\section{Subjects and data collection}

Data from 53 patients with 5q-SMA type 1, 2 or 3 treated with nusinersen (defined as at least one successful injection) in the Department of Neurology at the Essen University Hospital, Germany, were retrospectively analyzed.

Demographic data, clinical findings and patient history were obtained before therapy started. In addition, established motor assessments were performed and included the Revised Upper Limb Module (RULM) and the Hammersmith Functional Motor Scale Expanded (HFMSE).

Intrathecal therapy with nusinersen was carried out according to the recommended dosing schedule with four loading doses $(\mathrm{L})$ on treatment days $0(\mathrm{~L} 1=$ baseline $), 14$ (L2), 28 (L3), and 63 (L4) followed by maintenance doses (M) every four months (M1..., M8). Each dose comprised $12 \mathrm{mg}$ of nusinersen. Depending on the presence of scoliosis or spondylodesis, intrathecal injections were performed via conventional lumbar punctures on an outpatient basis or under image guidance (fluoroscopy-assisted or computed tomography (CT)-guided) during a short hospital stay. Prior to the injections, $5 \mathrm{~mL}$ of cerebrospinal fluid (CSF) was removed, and blood samples were collected for laboratory analyses according to our standard of care.

\section{CSF analyses}

Samples with a relevant increased red blood cell (RBC) count (defined as at least $1000 \mathrm{RBCs}$ per microliter) were excluded from statistical CSF analysis because of possible distortion of the other parameters. The following parameters were statistically evaluated: CSF white blood cell (CSF-WBC) count [number/ $\mu \mathrm{L}]$, glucose $[\mathrm{mg} / \mathrm{dL}]$, lactate $[\mathrm{mmol} / \mathrm{L}]$ and total protein $[\mathrm{mg} / \mathrm{dL}]$. The determination of CSF cell count was performed with the automated hematology analyzer XN 1000 (Sysmex Europe, Norderstedt, Germany) in body fluid mode using fluorescence flow cytometry. A distinction between mononuclear cells and granulocytes was performed in samples with pleocytosis. Glucose (hexokinase-glucose-6-phosphate dehydrogenase method), lactate (lactic dehydrogenase method) and total protein (pyrogallol red-molybdate method) were measured using a Dimension XPand clinical chemistry analyzer (Siemens Healthcare Diagnostics, Eschborn, Germany).

\section{Blood analyses}

Blood samples from outpatients were usually acquired before the first loading dose (L1) and each maintenance dose (M1 to M8). In contrast, in hospitalized patients, blood samples were generally obtained at each stay (L1 to L4 and M1 to M8).

The following parameters were collected from all patients and considered in the statistical evaluation: white blood cells (WBCs) and platelets from the blood count [number/nL] as well as creatinine $[\mathrm{mg} / \mathrm{dL}]$, urea nitrogen $[\mathrm{mg} / \mathrm{dL}]$, alanine aminotransferase (ALT) [U/L], aspartate aminotransferase (AST) $[\mathrm{U} / \mathrm{L}]$, gamma-glutamyltransferase (GGT) $[\mathrm{U} / \mathrm{L}]$ and C-reactive protein (CRP) $[\mathrm{mg} / \mathrm{dL}]$ from serum. Blood counts were obtained on an automated hematology analyzer XN 1000 (Sysmex Europe, Norderstedt, Germany) with impedance measurement and fluorescence flow cytometry. Clinical chemistry determinations were performed using Advia Chemistry and Atellica CH 930 analyzers (Siemens Healthcare Diagnostics, Eschborn, Germany). The creatinine assay is based on the Jaffe reaction using picric acid in an alkaline medium [19]. The activities of ALT and AST were measured with assays using pyridoxal-5'-phosphate and alpha-ketoglutarate as reagents. The GGT assay is based on a procedure according to Shaw [35]. The urea nitrogen assay is based on the Roch-Ramel enzymatic reaction using urease and glutamate dehydrogenase [34]. The CRP concentration was determined by an immunoturbidimetric assay.

In addition, before each injection, the activated partial thromboplastin time (aPTT) [s] and the international 
normalized ratio (INR) as a function of prothrombin time were measured in hospitalized patients as markers of the intrinsic and extrinsic pathways of coagulation, respectively. However, the coagulation values of outpatients were only measured and statistically evaluated in a few cases, as they were mostly collected in external laboratories with different reference values. The aPTT and prothrombin time were measured on CS-5100 analyzers (Sysmex Europe, Norderstedt, Germany) and Atellica COAG 360 (Siemens Healthcare Diagnostics, Eschborn, Germany) analyzers. The aPTT assay uses a soybean and rabbit brain phospholipid solution with a plasma activator with increased sensitivity to lupus anticoagulant-like substances. The prothrombin time assay uses thromboplastin and calcium as clotting activators. The INR is calculated using the lot and device-specific international sensitivity index value.

\section{Reference values}

The following reference values were used: CSF-WBC count $0-5 / \mu \mathrm{L}$, glucose $49-75 \mathrm{mg} / \mathrm{dL}$, lactate $1.2-2.1 \mathrm{mmol} / \mathrm{L}$, and total protein $15-45 \mathrm{mg} / \mathrm{dL}$. WBC count $3.6-9.2 / \mathrm{nL}$, platelet count $140-380 / \mathrm{nL}$, INR $0.85-1.15$, aPTT $24.4-32.4 \mathrm{~s}$, creatinine $0.6-1.3 \mathrm{mg} / \mathrm{dL}$, urea nitrogen $6.0-19.8 \mathrm{mg} / \mathrm{dL}$, AST and ALT $<50 \mathrm{U} / \mathrm{L}$ each, GGT $<55 \mathrm{U} / \mathrm{L}$, and $\mathrm{CRP}<0.5 \mathrm{mg} /$ $\mathrm{dL}$. It should be noted that some gender-specific reference values (platelet count as well as creatinine and liver values) were unified for the purpose of evaluation.

\section{Calculation of the mean change from baseline under therapy with nusinersen}

To identify trends in laboratory values in the total cohort and SMA subgroups on therapy, the mean change from baseline was calculated for each parameter. To increase the sensitivity for detecting longer-term trends, the last available measured value was compared with the respective baseline value in each patient. In addition, the mean change from baseline was calculated for each injection.

\section{Counting of deviations from the normal range under therapy with nusinersen}

To detect potential toxicities in individual patients, the number of patients with normal baseline values who showed deviations from the normal range under therapy (i.e., shifts to abnormal laboratory values) was counted. The duration of the deviations was divided into three categories: "Sporadic" deviations were defined as only single events with immediate normalization afterwards. "Transient" deviations were defined as two or more deviations in a row with subsequent normalization or two deviations in a row at the end of the observation period. "Persistent" deviations were defined as three or more deviations in a row without normalization until the end of the observation period. If patients met more than one criterion (e.g., a single and two consecutive elevations), they were assigned to the "worst" category. It should be noted that for aPTT and INR, only elevations were considered pathological (increased bleeding risk), while in CSF for lactate, only elevations, and for glucose, only decreases (possible signs of infection) were counted.

\section{Statistical analyses}

Statistical analyses were performed with SAS 9.4 software (SAS Institute Inc., Cary, North Carolina, United States) for Windows (Microsoft Corp., Redmond, Washington, United States). Continuous variables were described by the mean, median, standard deviation, quartiles and range. Categorical data were described by absolute and relative frequencies. The Mann-Whitney $U$ test or Kruskal-Wallis test was used for comparison of subgroups, and the sign test was used for the analysis of changes between injections. To analyze binary variables, Fisher's exact test was used. No adjustment for multiple testing was done. A two-sided $p$ value $<0.05$ was interpreted as significant. Because of the nature of the study, all statistical test results should be interpreted as explorative.

\section{Results}

\section{Subject and treatment characteristics}

From July 2017 to May 2020, a total of 53 adult SMA patients were treated with nusinersen (defined as at least one successful injection). Among these, two patients had SMA type 1, 15 patients had SMA type 2, and 36 patients had SMA type 3. SMA type 1 patients were not considered in the statistical analysis because of their small number and the limited set of data. Furthermore, one female SMA type 2 patient was excluded from analyses because the acquisition of CSF and administrations of nusinersen were performed using a port system with a spinal catheter. For detailed subject and treatment data, see Table 1 .

\section{Sample characteristics}

A full CSF data set (i.e., CSF-WBC count, total protein, glucose and lactate) was available from 50 of 50 baseline punctures $(100 \%)$ and from 391 of 404 punctures performed in total $(96.8 \%)$. In ten cases $(2.5 \%)$, CSF analyses were not feasible, which was due to insufficient sample volume. In two cases $(0.5 \%), \mathrm{CSF}$ analyses included only the WBC and RBC counts. Conversely, in one case $(0.2 \%)$, the cell count was missing. A total of 31 of 393 available 
Table 1 Subject and treatment characteristics

\begin{tabular}{|c|c|c|c|}
\hline & SMA type 2 & SMA type 3 & Total \\
\hline Number of patients & 14 & 36 & 50 \\
\hline Male & $5(35.7 \%)$ & $26(72.2 \%)$ & $31(62.0 \%)$ \\
\hline Female & $9(64.3 \%)$ & $10(27.8 \%)$ & $19(38.0 \%)$ \\
\hline Ambulatory & $0(0 \%)$ & $17(47.2 \%)$ & $17(34.0 \%)$ \\
\hline Spondylodesis & $7(50.0 \%)$ & $6(16.7 \%)$ & $13(26.0 \%)$ \\
\hline Age in years (mean $\pm \mathrm{SD})$ & $32.3 \pm 10.9$ & $37.8 \pm 12.9$ & $36.3 \pm 12.5$ \\
\hline Age in years (range) & $18-52$ & $18-71$ & $18-71$ \\
\hline HFMSE baseline score $($ mean \pm SD) & $2.4 \pm 2.2$ & $28.2 \pm 21.2$ & $21.0 \pm 21.4$ \\
\hline RULM baseline score (mean \pm SD) & $8.6 \pm 5.5$ & $25.7 \pm 12.1$ & $20.9 \pm 13.2$ \\
\hline Injection mode, Number of patients & 14 & 36 & 50 \\
\hline Conventional & $0(0 \%)$ & $23(63.9 \%)$ & $23(46.0 \%)$ \\
\hline Fluoroscopy & $0(0 \%)$ & $4(11.1 \%)$ & $4(8.0 \%)$ \\
\hline $\mathrm{CT}$ & $14(100 \%)$ & $9(25.0 \%)$ & $23(46.0 \%)$ \\
\hline Injection mode, Number of injections & 114 & 290 & 404 \\
\hline Conventional & $0(0 \%)$ & $195(67.2 \%)$ & $195(48.3 \%)$ \\
\hline Fluoroscopy & $0(0 \%)$ & $25(8.6 \%)$ & $25(6.2 \%)$ \\
\hline $\mathrm{CT}$ & $114(100 \%)$ & $70(24.1 \%)$ & $184(45.5 \%)$ \\
\hline \multicolumn{4}{|l|}{ Treatment duration } \\
\hline Patients with 12 injections $=34$ months & 0 & 2 & 2 \\
\hline Patients with 11 injections $=30$ months & 1 & 8 & 9 \\
\hline Patients with 10 injections $=26$ months & 3 & 4 & 7 \\
\hline Patients with 9 injections $=22$ months & 3 & 3 & 6 \\
\hline Patients with 8 injections $=18$ months & 3 & 1 & 4 \\
\hline Patients with 7 injections $=14$ months & 2 & 5 & 7 \\
\hline Patients with 6 injections $=10$ months & 1 & 5 & 6 \\
\hline Patients with 5 injections $=6$ months & 0 & 6 & 6 \\
\hline Patients with 4 injections $=2$ months & 0 & 2 & 2 \\
\hline Patients with 3 injections $=1$ month & 0 & 0 & 0 \\
\hline Patients with 2 injections $=14$ days & 1 & 0 & 1 \\
\hline Patients with 1 injection= baseline & 0 & 0 & 0 \\
\hline Total observation period (patient-years) & 22.2 & 54.7 & 76.9 \\
\hline
\end{tabular}

SMA spinal muscular atrophy, SD standard deviation, HFMSE Hammersmith Functional Motor Scale Expanded, RULM Revised Upper Limb Module, $C T$ computed tomography
CSF samples $(7.9 \%$, including three baseline samples) from 22 patients were excluded from statistical evaluation because of red blood cell contamination; thus, a total of 362 samples remained for further evaluation.

With regard to blood analyses, baseline values were available with the following frequencies: blood count (WBC and platelets) in 48 cases $(96.0 \%)$, kidney values (creatinine and urea nitrogen) and liver values (ALT, AST, and GGT) in 46 cases each $(92.0 \%)$, and CRP in 44 cases $(88.0 \%)$. Coagulation parameters (aPTT and INR) were found in 28 cases $(56.0 \%)$, as they were usually collected from hospitalized patients. The total number of available measurements was 327 for blood counts (80.9\%), 322 (urea nitrogen) and 323 (creatinine) for kidney values (79.7\% and $80.0 \%$, respectively), and 321 each for liver values and CRP (79.5\%). Coagulation parameters were available in 180 (aPTT) and 181 (INR) cases (44.6\% and $44.8 \%$, respectively).

\section{Laboratory analyses_-baseline values and changes during treatment with nusinersen}

Detailed results of baseline parameters (therapy-naïve patients $=\mathrm{L} 1$ ), including subgroup comparisons between SMA type 2 and type 3 , are provided in Table 2 and in the Supplemental Materials (Tables S1a-m). The results of the following injections (L2 to M8) are shown in Tables S1a-m. Mean changes from baseline for the total cohort and subgroups, including corresponding observation periods, are presented in Tables 3 and $\mathrm{S} 2 \mathrm{a}-\mathrm{m}$. The number of shifts to abnormal laboratory values in individual patients is shown in Table 4. 
Table 2 Baseline values with subgroup comparison (SMA type 2 versus SMA type 3 )

\begin{tabular}{|c|c|c|c|c|c|c|c|}
\hline & SMA & $\mathrm{n}_{\mathrm{BL}}$ & $\mathrm{n}_{\mathrm{BL}} \uparrow$ & $\mathrm{n}_{\mathrm{BL}} \downarrow$ & $\operatorname{Mean}^{\mathrm{a}} \pm \mathrm{SD}^{\mathrm{a}}$ & Range $^{\mathrm{a}}$ & $p$ value $^{\mathrm{b}}$ \\
\hline \multicolumn{8}{|l|}{ CSF } \\
\hline \multirow{3}{*}{$\begin{array}{l}\text { WBC } \\
(/ \mu \mathrm{L})\end{array}$} & 2 & $13^{\mathrm{c}}$ & 1 & $\mathrm{n} / \mathrm{a}$ & $2.3 \pm 4.3$ & $0-16$ & \\
\hline & 3 & $34^{\mathrm{c}}$ & 2 & $\mathrm{n} / \mathrm{a}$ & $2.4 \pm 2.4$ & $0-13$ & \\
\hline & Total & $47^{\mathrm{c}}$ & 3 & $\mathrm{n} / \mathrm{a}$ & $2.4 \pm 3.0$ & $0-16$ & 0.15 \\
\hline \multirow{3}{*}{$\begin{array}{l}\text { Glucose } \\
\text { (mg/dL) }\end{array}$} & 2 & $13^{\mathrm{c}}$ & $\mathrm{n} / \mathrm{a}$ & 0 & $62.5 \pm 5.8$ & $53-72$ & \\
\hline & 3 & $34^{\mathrm{c}}$ & $\mathrm{n} / \mathrm{a}$ & 0 & $65.7 \pm 5.4$ & $56-75$ & \\
\hline & Total & $47^{\mathrm{c}}$ & $\mathrm{n} / \mathrm{a}$ & 0 & $64.8 \pm 5.7$ & $53-75$ & 0.12 \\
\hline \multirow{3}{*}{$\begin{array}{l}\text { Lactate } \\
(\mathrm{mmol} / \mathrm{L})\end{array}$} & 2 & $13^{\mathrm{c}}$ & 0 & $\mathrm{n} / \mathrm{a}$ & $1.35 \pm 0.19$ & $1.0-1.7$ & \\
\hline & 3 & $34^{\mathrm{c}}$ & 1 & $\mathrm{n} / \mathrm{a}$ & $1.57 \pm 0.27$ & $1.1-2.5$ & \\
\hline & Total & $47^{\mathrm{c}}$ & 1 & $\mathrm{n} / \mathrm{a}$ & $1.51 \pm 0.27$ & $1.0-2.5$ & 0.01 \\
\hline \multirow{3}{*}{$\begin{array}{l}\text { Protein } \\
\text { (mg/dL) }\end{array}$} & 2 & $13^{\mathrm{c}}$ & 1 & 0 & $34.1 \pm 8.6$ & $19-46$ & \\
\hline & 3 & $34^{\mathrm{c}}$ & 11 & 0 & $41.9 \pm 12.4$ & $26-72$ & \\
\hline & Total & $47^{\mathrm{c}}$ & 12 & 0 & $39.7 \pm 11.9$ & $19-72$ & 0.07 \\
\hline \multicolumn{8}{|l|}{ Blood } \\
\hline \multirow{3}{*}{$\begin{array}{l}\text { WBC } \\
(/ \mathrm{nL})\end{array}$} & 2 & 13 & 2 & 0 & $7.2 \pm 2.2$ & $3.9-11.7$ & \\
\hline & 3 & 35 & 5 & 0 & $7.1 \pm 1.8$ & $4.6-11.9$ & \\
\hline & Total & 48 & 7 & 0 & $7.1 \pm 1.9$ & $3.9-11.9$ & 0.94 \\
\hline \multirow{3}{*}{$\begin{array}{l}\text { CRP } \\
(\mathrm{mg} / \mathrm{dL})\end{array}$} & 2 & 13 & 5 & $\mathrm{n} / \mathrm{a}$ & - & $<0.5-1.5$ & \\
\hline & 3 & 31 & 10 & $\mathrm{n} / \mathrm{a}$ & - & $<0.5-2.3$ & \\
\hline & Total & 44 & 15 & $\mathrm{n} / \mathrm{a}$ & - & $<0.5-2.3$ & 0.68 \\
\hline \multirow{3}{*}{$\begin{array}{l}\text { Platelets } \\
\text { (/nL) }\end{array}$} & 2 & 13 & 5 & 2 & $315.2 \pm 122.0$ & $103-477$ & \\
\hline & 3 & 35 & 0 & 0 & $265.9 \pm 50.9$ & $176-368$ & \\
\hline & Total & 48 & 5 & 2 & $279.3 \pm 78.5$ & $103-477$ & 0.07 \\
\hline \multirow[t]{3}{*}{ INR } & 2 & 12 & 0 & $\mathrm{n} / \mathrm{a}$ & $1.02 \pm 0.06$ & $0.92-1.12$ & \\
\hline & 3 & 16 & 1 & $\mathrm{n} / \mathrm{a}$ & $1.02 \pm 0.08$ & $0.93-1.19$ & \\
\hline & Total & 28 & 1 & $\mathrm{n} / \mathrm{a}$ & $1.02 \pm 0.07$ & $0.92-1.19$ & 0.96 \\
\hline \multirow{3}{*}{$\begin{array}{l}\text { aPTT } \\
\text { (s) }\end{array}$} & 2 & 12 & 0 & $\mathrm{n} / \mathrm{a}$ & $28.8 \pm 2.6$ & $24.3-32.1$ & \\
\hline & 3 & 16 & 1 & $\mathrm{n} / \mathrm{a}$ & $27.5 \pm 3.1$ & $20.9-32.9$ & \\
\hline & Total & 28 & 1 & $\mathrm{n} / \mathrm{a}$ & $28.1 \pm 2.9$ & $20.9-32.9$ & 0.29 \\
\hline \multirow{3}{*}{$\begin{array}{l}\text { Crea } \\
(\mathrm{mg} / \mathrm{dL})\end{array}$} & 2 & 13 & 0 & 13 & $0.31 \pm 0.05$ & $0.26-0.43$ & \\
\hline & 3 & 33 & 0 & 24 & $0.49 \pm 0.14$ & $0.28-0.78$ & \\
\hline & Total & 46 & 0 & 37 & $0.44 \pm 0.15$ & $0.26-0.78$ & 0.0001 \\
\hline \multirow{3}{*}{$\begin{array}{l}\text { Urea } \\
(\mathrm{mg} / \mathrm{dL})\end{array}$} & 2 & 13 & 0 & 2 & $8.8 \pm 4.1$ & $4.0-18.0$ & \\
\hline & 3 & 33 & 3 & 1 & $11.9 \pm 4.3$ & $5.0-22.0$ & \\
\hline & Total & 46 & 3 & 3 & $11.0 \pm 4.5$ & $4.0-22.0$ & 0.02 \\
\hline \multirow{3}{*}{$\begin{array}{c}\text { AST } \\
(\mathrm{U} / \mathrm{L})\end{array}$} & 2 & 13 & 0 & $\mathrm{n} / \mathrm{a}$ & $21.0 \pm 8.9$ & $11-39$ & \\
\hline & 3 & 33 & 1 & $\mathrm{n} / \mathrm{a}$ & $28.5 \pm 13.1$ & $11-72$ & \\
\hline & Total & 46 & 1 & $\mathrm{n} / \mathrm{a}$ & $26.4 \pm 12.4$ & $11-72$ & 0.07 \\
\hline \multirow{3}{*}{$\begin{array}{c}\text { ALT } \\
(\mathrm{U} / \mathrm{L})\end{array}$} & 2 & 13 & 2 & $\mathrm{n} / \mathrm{a}$ & $31.5 \pm 29.1$ & $8-111$ & \\
\hline & 3 & 33 & 14 & $\mathrm{n} / \mathrm{a}$ & $47.7 \pm 24.2$ & $17-118$ & \\
\hline & Total & 46 & 16 & $\mathrm{n} / \mathrm{a}$ & $43.2 \pm 26.4$ & $8-118$ & 0.01 \\
\hline \multirow{3}{*}{$\begin{array}{r}\text { GGT } \\
(\mathrm{U} / \mathrm{L})\end{array}$} & 2 & 13 & 2 & $\mathrm{n} / \mathrm{a}$ & $36.1 \pm 45.1$ & $7-176$ & \\
\hline & 3 & 33 & 7 & $\mathrm{n} / \mathrm{a}$ & $43.7 \pm 39.3$ & $7-190$ & \\
\hline & Total & 46 & 9 & $\mathrm{n} / \mathrm{a}$ & $41.5 \pm 40.7$ & $7-190$ & 0.14 \\
\hline
\end{tabular}

CSF cerebrospinal fluid, SMA spinal muscular atrophy, WBC white blood cell count, CRP C-reactive protein, INR international normalized ratio, aPTT activated partial thromboplastin time, crea creatinine, urea urea nitrogen, $A S T$ aspartate aminotransferase, $A L T$ alanine aminotransferase, $G G T$ gamma-glutamyltransferase, $n_{B L}$ number of available baseline values, $n_{B L} \uparrow$ number of elevated baseline values, $n_{B L} \downarrow$ number of lowered baseline values, $S D$ standard deviation, $n / a$ not applicable (only medically reasonable or meaningful directions of deviation were considered)

${ }^{\mathrm{a}}$ Units as specified in the first column

${ }^{\mathrm{b}}$ SMA type 2 versus SMA type 3

${ }^{\mathrm{c}}$ Three CSF baseline samples were excluded from statistical analyses because of red blood cell contamination 
Table 3 Mean change from baseline under therapy (last available versus baseline values)

\begin{tabular}{|c|c|c|c|c|c|c|}
\hline & SMA & $N$ & $\begin{array}{l}\text { Observ. period } \\
\text { Mean (months) }\end{array}$ & $\begin{array}{l}\text { Baseline } \\
\text { Mean }^{\mathrm{a}}\end{array}$ & $\begin{array}{l}\text { Change } \\
\text { Mean }^{\mathrm{a}}\end{array}$ & $p$ value \\
\hline \multicolumn{7}{|l|}{$\mathrm{CSF}$} \\
\hline \multirow{3}{*}{$\begin{array}{l}\text { WBC } \\
(/ \mu \mathrm{L})\end{array}$} & 2 & 12 & 20.7 & 2.4 & +0.2 & 0.51 \\
\hline & 3 & 34 & 17.8 & 2.4 & -0.4 & 0.50 \\
\hline & Total & 46 & 18.5 & 2.4 & -0.2 & 1.00 \\
\hline \multirow{3}{*}{$\begin{array}{l}\text { Glucose } \\
(\mathrm{mg} / \mathrm{dL})\end{array}$} & 2 & 12 & 20.7 & 62.6 & -1.7 & 0.75 \\
\hline & 3 & 34 & 17.6 & 65.7 & -2.1 & 0.04 \\
\hline & Total & 46 & 18.4 & 64.9 & -2.0 & 0.04 \\
\hline \multirow{3}{*}{$\begin{array}{l}\text { Lactate } \\
(\mathrm{mmol} / \mathrm{L})\end{array}$} & 2 & 12 & 20.7 & 1.33 & +0.10 & 0.18 \\
\hline & 3 & 34 & 17.6 & 1.57 & +0.01 & 0.69 \\
\hline & Total & 46 & 18.4 & 1.51 & +0.03 & 0.23 \\
\hline \multirow{3}{*}{$\begin{array}{l}\text { Protein } \\
(\mathrm{mg} / \mathrm{dL})\end{array}$} & 2 & 12 & 20.7 & 33.2 & +3.8 & 0.75 \\
\hline & 3 & 34 & 17.6 & 41.9 & +2.6 & 0.01 \\
\hline & Total & 46 & 18.4 & 39.6 & +2.9 & 0.01 \\
\hline \multicolumn{7}{|l|}{ Blood } \\
\hline \multirow{3}{*}{$\begin{array}{l}\text { WBC } \\
(/ \mathrm{nL})\end{array}$} & 2 & 13 & 17.3 & 7.2 & +0.1 & 0.58 \\
\hline & 3 & 35 & 17.4 & 7.1 & -0.2 & 0.74 \\
\hline & Total & 48 & 17.4 & 7.1 & -0.1 & 1.00 \\
\hline \multirow{3}{*}{$\begin{array}{l}\text { Platelets } \\
(/ \mathrm{nL})\end{array}$} & 2 & 13 & 17.3 & 315.2 & -39.0 & 0.27 \\
\hline & 3 & 35 & 17.4 & 265.9 & +2.7 & 0.86 \\
\hline & Total & 48 & 17.4 & 279.3 & -8.6 & 0.77 \\
\hline \multirow[t]{3}{*}{ INR } & 2 & 12 & 18.2 & 1.02 & +0.01 & 0.75 \\
\hline & 3 & 13 & 12.0 & 1.03 & +0.01 & 1.00 \\
\hline & Total & 25 & 15.0 & 1.02 & +0.01 & 0.83 \\
\hline \multirow{3}{*}{$\begin{array}{l}\text { aPTT } \\
\text { (s) }\end{array}$} & 2 & 12 & 18.2 & 28.8 & -2.1 & 0.04 \\
\hline & 3 & 13 & 12.0 & 28.1 & -0.7 & 0.09 \\
\hline & Total & 25 & 15.0 & 28.4 & -1.4 & 0.004 \\
\hline \multirow{3}{*}{$\begin{array}{l}\text { Crea } \\
(\mathrm{mg} / \mathrm{dL})\end{array}$} & 2 & 13 & 16.7 & 0.31 & -0.04 & 0.15 \\
\hline & 3 & 33 & 17.9 & 0.49 & -0.11 & $<0.0001$ \\
\hline & Total & 46 & 17.5 & 0.44 & -0.09 & $<0.0001$ \\
\hline \multirow{3}{*}{$\begin{array}{l}\text { Urea } \\
(\mathrm{mg} / \mathrm{dL})\end{array}$} & 2 & 13 & 16.7 & 8.8 & +1.5 & 0.27 \\
\hline & 3 & 33 & 17.8 & 11.9 & -0.5 & 0.58 \\
\hline & Total & 46 & 17.4 & 11.0 & +0.0 & 1.00 \\
\hline \multirow{3}{*}{$\begin{array}{c}\text { AST } \\
(\mathrm{U} / \mathrm{L})\end{array}$} & 2 & 13 & 16.3 & 21.0 & -1.6 & 0.09 \\
\hline & 3 & 33 & 17.8 & 28.5 & +1.2 & 1.00 \\
\hline & Total & 46 & 17.4 & 26.4 & +0.4 & 0.46 \\
\hline \multirow{3}{*}{$\begin{array}{l}\text { ALT } \\
(\mathrm{U} / \mathrm{L})\end{array}$} & 2 & 13 & 16.3 & 31.5 & -4.7 & 1.00 \\
\hline & 3 & 33 & 17.8 & 47.7 & -1.6 & 0.22 \\
\hline & Total & 46 & 17.4 & 43.2 & -2.5 & 0.36 \\
\hline \multirow{3}{*}{$\begin{array}{l}\text { GGT } \\
(\mathrm{U} / \mathrm{L})\end{array}$} & 2 & 13 & 16.3 & 36.1 & +3.5 & 1.00 \\
\hline & 3 & 33 & 17.8 & 43.7 & -6.2 & 0.49 \\
\hline & Total & 46 & 17.4 & 41.5 & -3.5 & 0.46 \\
\hline
\end{tabular}

CSF cerebrospinal fluid, SMA spinal muscular atrophy, WBC white blood cell count, INR international normalized ratio, aPTT activated partial thromboplastin time, crea creatinine, urea urea nitrogen, $A S T$ aspartate aminotransferase, $A L T$ alanine aminotransferase, GGT gamma-glutamyltransferase, $n$ number of patients with at least one baseline and one follow-up sample, observ. period observation period

${ }^{a}$ Units as specified in the first column

${ }^{\mathrm{b}}$ For each patient, the last measured value was compared with the respective baseline value 
Table 4 Number of patients with shifts to abnormal laboratory values under therapy

\begin{tabular}{|c|c|c|c|c|c|c|}
\hline & \multirow{2}{*}{$\begin{array}{l}\text { Baseline } \\
\text { Normal }\end{array}$} & \multicolumn{5}{|c|}{ Deviation from normal range under therapy } \\
\hline & & Dir & Sporadic $^{\mathrm{a}}$ & Transient $^{\mathrm{b}}$ & Persistent $^{\mathrm{c}}$ & No deviation \\
\hline \multicolumn{7}{|l|}{ CSF } \\
\hline WBC & 44 & 1 & $\begin{array}{l}7(15.9 \%) \\
\mathrm{n} / \mathrm{a}\end{array}$ & $\begin{array}{l}2(4.5 \%) \\
\mathrm{n} / \mathrm{a}\end{array}$ & $\begin{array}{l}0(0.0 \%) \\
\mathrm{n} / \mathrm{a}\end{array}$ & $35(79.5 \%)$ \\
\hline Glucose & 47 & 1 & $\begin{array}{l}\mathrm{n} / \mathrm{a} \\
0(0.0 \%)\end{array}$ & $\begin{array}{l}\mathrm{n} / \mathrm{a} \\
0(0.0 \%)\end{array}$ & $\begin{array}{l}\mathrm{n} / \mathrm{a} \\
0(0.0 \%)\end{array}$ & $47(100 \%)$ \\
\hline Lactate & 46 & 1 & $\begin{array}{l}3(6.5 \%) \\
\text { n/a }\end{array}$ & $\begin{array}{l}1(2.2 \%) \\
\mathrm{n} / \mathrm{a}\end{array}$ & $\begin{array}{l}0(0.0 \%) \\
\mathrm{n} / \mathrm{a}\end{array}$ & $42(91.3 \%)$ \\
\hline Protein & 35 & 1 & $\begin{array}{l}6(17.1 \%) \\
\text { n/a }\end{array}$ & $\begin{array}{l}3(8.6 \%) \\
\text { n/a }\end{array}$ & $\begin{array}{l}2(5.7 \%) \\
\mathrm{n} / \mathrm{a}\end{array}$ & $24(68.6 \%)$ \\
\hline \multicolumn{7}{|l|}{ Blood } \\
\hline WBC & 41 & 1 & $\begin{array}{l}7(17.1 \%) \\
1(2.4 \%)\end{array}$ & $\begin{array}{l}0(0.0 \%) \\
0(0.0 \%)\end{array}$ & $\begin{array}{l}1(2.4 \%) \\
0(0.0 \%)\end{array}$ & $32(78.0 \%)$ \\
\hline CRP & 29 & 1 & $\begin{array}{l}9(31.0 \%) \\
\mathrm{n} / \mathrm{a}\end{array}$ & $\begin{array}{l}3(10.3 \%) \\
\text { n/a }\end{array}$ & $\begin{array}{l}0(0.0 \%) \\
\mathrm{n} / \mathrm{a}\end{array}$ & $17(58.6 \%)$ \\
\hline Platelets & 41 & 1 & $\begin{array}{l}1(2.4 \%) \\
1(2.4 \%)\end{array}$ & $\begin{array}{l}1(2.4 \%) \\
0(0.0 \%)\end{array}$ & $\begin{array}{l}0(0.0 \%) \\
0(0.0 \%)\end{array}$ & $38(92.7 \%)$ \\
\hline INR & 27 & 1 & $\begin{array}{l}2(7.4 \%) \\
\text { n/a }\end{array}$ & $\begin{array}{l}0(0.0 \%) \\
\mathrm{n} / \mathrm{a}\end{array}$ & $\begin{array}{l}0(0.0 \%) \\
\mathrm{n} / \mathrm{a}\end{array}$ & $25(92.6 \%)$ \\
\hline aPTT & 27 & 1 & $\begin{array}{l}4(14.8 \%) \\
\text { n/a }\end{array}$ & $\begin{array}{l}0(0.0 \%) \\
\mathrm{n} / \mathrm{a}\end{array}$ & $\begin{array}{l}0(0.0 \%) \\
\mathrm{n} / \mathrm{a}\end{array}$ & $23(85.2 \%)$ \\
\hline Crea & 9 & 1 & $\begin{array}{l}0(0.0 \%) \\
2(22.2 \%)\end{array}$ & $\begin{array}{l}0(0.0 \%) \\
3(33.3 \%)\end{array}$ & $\begin{array}{l}0(0.0 \%) \\
1(11.1 \%)\end{array}$ & $3(33.3 \%)$ \\
\hline Urea & 40 & 1 & $\begin{array}{l}3(7.5 \%) \\
3(7.5 \%)\end{array}$ & $\begin{array}{l}0(0.0 \%) \\
0(0.0 \%)\end{array}$ & $\begin{array}{l}0(0.0 \%) \\
0(0.0 \%)\end{array}$ & $34(85.0 \%)$ \\
\hline AST & 45 & 1 & $\begin{array}{l}2(4.4 \%) \\
\mathrm{n} / \mathrm{a}\end{array}$ & $\begin{array}{l}1(2.2 \%) \\
\mathrm{n} / \mathrm{a}\end{array}$ & $\begin{array}{l}1(2.2 \%) \\
\mathrm{n} / \mathrm{a}\end{array}$ & $41(91.1 \%)$ \\
\hline ALT & 30 & 1 & $\begin{array}{l}2(6.7 \%) \\
\mathrm{n} / \mathrm{a}\end{array}$ & $\begin{array}{l}1(3.3 \%) \\
\mathrm{n} / \mathrm{a}\end{array}$ & $\begin{array}{l}0(0.0 \%) \\
\mathrm{n} / \mathrm{a}\end{array}$ & $27(90.0 \%)$ \\
\hline GGT & 37 & 1 & $\begin{array}{l}0(0.0 \%) \\
\mathrm{n} / \mathrm{a}\end{array}$ & $\begin{array}{l}2(5.4 \%) \\
\mathrm{n} / \mathrm{a}\end{array}$ & $\begin{array}{l}1(2.7 \%) \\
\mathrm{n} / \mathrm{a}\end{array}$ & $34(91.9 \%)$ \\
\hline
\end{tabular}

$C S F$ cerebrospinal fluid, WBC white blood cell count, CRP C-reactive protein, INR international normalized ratio, aPTT activated partial thromboplastin time, crea creatinine, urea urea nitrogen, AST aspartate aminotransferase, ALT alanine aminotransferase, GGT gamma-glutamyltransferase, Dir direction, ,

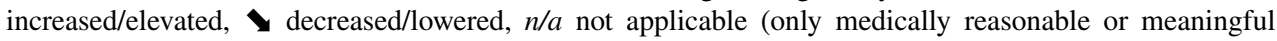
directions of deviation were considered)

${ }^{a}$ Only singular deviations from the normal range with immediate normalization afterwards

${ }^{b}$ Two or more deviations in a row with normalization afterwards or two deviations in a row at the end of the observation period

${ }^{\mathrm{c}}$ Three or more deviations in a row without normalization until the end of the observation period

\section{CSF parameters}

At baseline, the mean CSF-WBC count was $2.4 / \mu \mathrm{L}$, with no difference between SMA type 2 and type 3 subgroups. Pleocytosis was seen in three cases $(6.4 \%)$. One SMA type 2 patient with spondylodesis, a restrictive ventilation disorder with documented recurrent pneumonias, and noninvasive ventilation at night showed pleocytosis of $16 / \mu \mathrm{L}(14 /$ $\mu \mathrm{L}$ mononuclear cells, $2 / \mu \mathrm{L}$ granulocytes). In another patient with SMA type 3 and spondylodesis, the CSF-WBC count was $13 / \mu \mathrm{L}$, and all of them were granulocytes. The third case was an SMA type 3 patient with a CSF-WBC count of 6/ $\mu \mathrm{L}(5 / \mu \mathrm{L}$ mononuclear cells, $1 / \mu \mathrm{L}$ granulocytes $)$. However, none of these patients had any clinical evidence of infectious or noninfectious CNS inflammation. Under therapy with nusinersen, the mean CSF-WBC count remained stable over time. However, during the first three injections, there was a trend towards higher CSF-WBC counts $(p=0.05)$. From 44 subjects with normal CSF-WBC counts at baseline, nine showed sporadic or transient elevations up to $21 / \mu \mathrm{L}$ during the observation. No patient developed persistent pleocytosis.

Glucose levels at baseline were normal in all patients, with no subgroup differences. Mean glucose levels decreased by $2 \mathrm{mg} / \mathrm{dL}(p=0.04)$. Since CSF glucose levels 
are associated with blood glucose levels, this slight change was not considered clinically relevant. In addition, no shifts to abnormal glucose levels were observed during therapy.

Mean lactate levels at baseline were normal but lower in SMA type 2 patients than in SMA type 3 patients $(p=0.01)$. In a single SMA type 3 patient, lactate was slightly elevated to $2.5 \mathrm{mmol} / \mathrm{L}$ at the first injection. In the same patient, total protein was elevated to $68 \mathrm{mg} / \mathrm{dL}$, indicating a BBB disturbance, whereas CSF-WBC count and glucose were normal. In general, mean lactate levels remained stable during the course of therapy. Three patients showed a sporadic elevation of lactate, while one patient had slightly elevated lactate levels up to $2.3 \mathrm{mmol} / \mathrm{L}$ in several samples, two of them consecutively. Analogous to the case mentioned above, this patient already showed a high-normal baseline value of $2.1 \mathrm{mmol} / \mathrm{L}$ and elevated total protein levels, probably indicating a BBB disorder.

In therapy-naïve patients, total protein was in the upper normal range with a mean value of $39.7 \mathrm{mg} / \mathrm{dL}$ and a trend towards higher levels in SMA type 3 patients $(p=0.07)$. Normal values were obtained from 35 patients. In eleven SMA type 3 patients, elevations up to $72 \mathrm{mg} / \mathrm{dL}$ were observed before therapy started. In contrast, there was only one SMA type 2 patient with a marginal elevation to $46 \mathrm{mg} / \mathrm{dL}$. Under therapy, the total cohort showed an increase to $42.5 \mathrm{mg} / \mathrm{dL}$ $(p=0.01)$. In the subgroup analysis, total protein increased by $3.8 \mathrm{mg} / \mathrm{dL}$ in SMA type 2 and $2.6 \mathrm{mg} / \mathrm{dL}$ in SMA type 3 patients. However, this difference was significant only in SMA type $3(p=0.01)$. Nine patients with normal baseline values showed sporadic or transient increases up to $76 \mathrm{mg} /$ $\mathrm{dL}$ during therapy. A persistent increase was observed in two patients (5.7\%): One SMA type 3 patient with a high-normal baseline of $43 \mathrm{mg} / \mathrm{dL}$ developed values up to $61 \mathrm{mg} / \mathrm{dL}$ from M3 to M6 (end of the observation period). In addition, a single SMA type 2 patient with a baseline value of $36 \mathrm{mg} /$ $\mathrm{dL}$ developed elevated total protein levels up to $67 \mathrm{mg} / \mathrm{dL}$ from M2 to M5 (end of observation). In both patients, the other CSF parameters showed no alterations.

\section{Inflammatory markers}

The WBC count was elevated in 7 of 48 available baseline samples with a maximum of $11.9 / \mathrm{nL}$. The CRP was slightly elevated in 15 of 44 baseline samples with a maximum of $2.3 \mathrm{mg} / \mathrm{dL}$. For both parameters, there were no differences between SMA type 2 and SMA type 3 patients. Mean WBC counts remained stable under therapy with nusinersen. Most subjects showed no or only slight sporadic increases in the WBC count up to $11.9 / \mathrm{nL}$. A single SMA type 2 patient (2.4\%) showed mild leukocytosis up to $11.1 / \mathrm{nL}$ in the last three available measurements. In the same patient, CRP levels were slightly increased up to $0.8 \mathrm{mg} / \mathrm{dL}$, possibly indicating mild systemic inflammation.

\section{Platelets}

At baseline, mean platelet levels were normal, and platelet count abnormalities were only observed in SMA type 2 patients. The platelet count was lowered in two patients with a minimum value of $103 / \mathrm{nL}$, whereas thrombocytosis was observed in five subjects with a maximum of $477 / \mathrm{nL}$. During therapy with nusinersen, there was no evidence of a change in the mean platelet count, either between the first and last injections or during the loading dosing. Analyses of individual patients revealed only a few shifts to abnormal values. Of 41 patients with normal platelet levels at baseline, a single SMA type 2 patient showed sporadic mild thrombocytopenia of $101 / \mathrm{nL}$ at M4, although dilution of the blood count cannot be ruled out, as leukocytes were also lower than usual. Furthermore, one SMA type 3 patient showed transient thrombocytosis up to $532 / \mathrm{nL}$ at M1/M2 with subsequent normalization (M3-M6). Another SMA type 3 patient showed singular thrombocytosis of $422 / \mathrm{nL}$ at M1.

\section{Coagulation parameters}

Baseline coagulation values were normal in most patients. Only one SMA type 3 patient showed a marginal prolonged aPTT to $32.9 \mathrm{~s}$, whereas another SMA type 3 patient showed a slight increase in INR to 1.19. No change of mean INR was observed over time. In contrast, the mean aPTT decreased by $1.4 \mathrm{~s}$ in the total cohort $(p=0.004)$ and $2.1 \mathrm{~s}$ in SMA type 2 patients $(p=0.04)$. During the observation period, there were only a few and slight sporadic increases in aPTT and INR to a maximum of $33.3 \mathrm{~s}$ and 1.18 , respectively.

\section{Kidney values}

In therapy-naïve patients, creatinine levels were lowered in all SMA type 2 patients and in 24 of 33 SMA type 3 patients. Remarkably, the mean values of both subgroups were below the normal range ( 0.31 and $0.49 \mathrm{mg} / \mathrm{dL}$, respectively). Furthermore, SMA type 2 patients had significantly lower creatinine values than SMA type 3 patients $(p=0.0001)$. Mean urea nitrogen levels were normal, with higher levels found in SMA type 3 patients $(p=0.02)$. In three patients each, urea nitrogen was slightly decreased or increased. During the observation period, the mean creatinine levels decreased by $0.09 \mathrm{mg} / \mathrm{dL}$ in the total cohort $(p<0.0001)$, while a decrease of $0.11 \mathrm{mg} / \mathrm{dL}$ was found in SMA type 3 patients $(p<0.0001)$. In contrast, the mean urea nitrogen levels remained unchanged over time. Of nine SMA type 3 patients with normal baseline values, six developed lowered creatinine levels during the course of therapy, with one decrease being persistent (11.1\%). Urea nitrogen levels changed only sporadically and mildly in a few cases, with a minimum of $5 \mathrm{mg} / \mathrm{dL}$ and a maximum of $23 \mathrm{mg} / \mathrm{dL}$. 


\section{Liver values}

At baseline, mean AST levels were normal, with SMA type 3 patients tending to have higher values than type 2 patients $(p=0.07)$. An elevated AST to $72 \mathrm{U} / \mathrm{L}$ was observed in a single SMA type 3 patient. In comparison, ALT and GGT were more frequently elevated (16 and 9 cases with a maximum of 118 and $190 \mathrm{U} / \mathrm{L}$, respectively). Subgroup comparison showed higher ALT levels in SMA type 3 patients $(p=0.01)$, whereas for GGT, there was no difference between SMA types. Under therapy, analysis of mean enzyme levels revealed no relevant changes in either the total cohort or the SMA subgroups. In individual patients, few and mainly sporadic or transient elevations were observed. However, one SMA type 3 patient (2.2\%) developed mild but persistent elevated AST values up to $58 \mathrm{U} / \mathrm{L}$, while one SMA type 2 patient $(2.7 \%)$ showed a successive and persistent increase in GGT up to $101 \mathrm{U} / \mathrm{L}$ from M3 to M5 (end of observation).

\section{Influence of the injection method on laboratory values}

A possible relationship between the injection method (conventional vs. image-guided) and the change in laboratory values from baseline was investigated. In summary, there was no evidence that the injection method had any influence on the development of laboratory values under therapy (data not shown).

\section{Discussion}

Laboratory parameters can help to identify side effects and organ toxicities of new therapies and thus to provide information on therapy safety. In the past, a variety of side effects have been identified for different ASOs, such as platelet declines, renal impairment, increases in liver enzymes or prolongation of aPTT $[3,7,15,20]$. In particular, thrombocytopenia, including acute severe thrombocytopenia, and nephrotoxicity are major concerns and are often considered class effects. However, these side effects seem to occur only with the use of some ASOs [8-10, 15]. Since data on laboratory findings in adult SMA patients under therapy with nusinersen are lacking, the present study helps to assess the safety of the therapy better.

Analysis of CSF parameters can detect various pathologies, such as infectious or noninfectious CNS inflammation, intrathecal hemorrhage, changes in CSF flow, or BBB dysfunction. Changes in CSF parameters under therapy with nusinersen are of particular interest since the drug has to be administered by repeated lumbar punctures. In our cohort, the mean baseline values of the analyzed CSF parameters were all within the normal range. Three patients showed mild pleocytosis at baseline; however, an association with
SMA is unlikely. In addition, the mean CSF-WBC count tended to increase during the first three injections (L1-L3). Furthermore, approximately half of the CSF samples with pleocytosis were obtained at L2 or L3. In line with our results, Müschen et al. and Wurster et al. found a transient increase in mean CSF-WBC counts during loading dosing. Also in these studies, all increases in CSF-WBC counts (four increases in three of 88 SMA patients) occurred at L2 or L3 $[32,41]$. These observations are most likely a reaction to the rapid succession of lumbar punctures at the beginning of the therapy. Under therapy, we found mild sporadic or transient elevations in CSF-WBC counts in approximately one-fifth of the patients, whereas no persistent increase was seen. In contrast, Müschen et al., Wurster et al. and Kessler et al. observed only three cases of pleocytosis in 28, 60 and 10 SMA patients treated for a period of 2 to 18 months [21, 32, 41].

Total CSF protein, an indicator of BBB and CSF flow integrity, was elevated in eleven SMA type 3 patients but only in one SMA type 2 patient. However, the widely used upper limit of normal (ULN) of $45 \mathrm{mg} / \mathrm{dL}$ is now being challenged [5,6]. Although significance was not reached in our cohort, the trend of higher CSF protein concentrations in SMA type 3 patients is consistent with the findings of Müschen et al. and Wurster et al. [32, 41]. Since total protein increases with age, the difference in patient age between the SMA type 2 and type 3 subgroups could be an explanation although it was only 5.5 years in our cohort [27]. During nusinersen therapy, two patients in our cohort developed a sustained elevation of total protein. Furthermore, mean protein levels increased significantly in the total cohort as well as in the SMA type 3 subgroup. These findings are compatible with results from other studies on nusinersen in adult patients and may indicate an alteration of CSF flow or BBB dysfunction. Whether this observation is a consequence of repeated lumbar punctures or a side effect of the drug remains unclear [21, 32, 39, 41]. This outcome may possibly be related to reports of rare cases of hydrocephalus of unknown etiology.

Recently, some cases of severe thrombocytopenia with platelet counts $<25 / \mathrm{nL}$ have been reported in clinical trials on ASOs. In the NEURO-TTR trial, three cases (3\%) of severe thrombocytopenia, including one in association with a fatal intracranial hemorrhage, were observed during therapy with inotersen, an ASO designed to inhibit hepatic production of transthyretin in patients with hereditary transthyretin amyloidosis. In the same study, a decrease in platelet count to less than $140 / \mathrm{nL}$ was seen in $54 \%$ of the participants in the inotersen group vs. $13 \%$ in the placebo group [4]. In another study, severe thrombocytopenia was found in 2 of 33 patients with familial chylomicronemia treated with volanesorsen [40]. Based on experience with other ASOs, thrombocytopenia, including acute severe thrombocytopenia, is part of 
the warnings in the prescribing information of nusinersen. Data on platelet counts under therapy with nusinersen are mainly available from studies in infants or children. In the ENDEAR and CHERISH studies, the incidence of shifts to low platelet counts was 13 vs. $0 \%$ (nusinersen vs. placebo) and 20 vs. $26 \%$, respectively. Platelet counts $<20 /$ $\mu \mathrm{L}$ were seen in one infant in the ENDEAR study and in two children in the CHERISH study. However, no sustained shifts or bleeding complications occurred. Median platelet counts remained stable during therapy [11]. Another study of nusinersen therapy in presymptomatic infants also showed stable platelet counts [12]. In a cohort of 58 SMA patients aged between 1 month and 56 years, Goedeker et al. described three cases of mild thrombocytopenia, with none of them reaching a platelet count $<100 / \mathrm{nL}[17]$. Szabo et al. reported singular mild and transient thrombocytopenia in a single child treated with nusinersen [38]. Remarkably, only a single case of sporadic thrombocytopenia with a platelet count of $101 / \mathrm{nL}$ occurred in our study. For the graduation of abnormal laboratory values, National Cancer Institute-Common Terminology Criteria of Adverse Events (NCI-CTCAE version 5.0) is often used in clinical studies, which distinguishes between four severity grades (grade 1, mild, to grade 4, severe) [1]. According to this reference, the observed thrombocytopenia would meet the criterion of grade 1 thrombocytopenia, defined as a platelet count between the lower limit of normal (LLN) and 75/nL. Similar to the data from pediatric studies, platelet levels remained stable over time. Based on our results, there is currently no evidence of an increased risk of severe thrombocytopenia in adult patients treated with nusinersen.

We observed a reduction in the mean aPTT between the first and last injections in the total cohort and the SMA type 2 subgroup. Shortened aPTTs are often considered laboratory artifacts, which are due to difficult blood sampling. In addition, there is some evidence that short aPTTs represent a hypercoagulable state and may be associated with an increased risk for thromboembolic and cardiovascular events $[23,30]$. With regard to the coagulation parameters, we did not observe any persistent parameter deviations from the normal range, which is in line with the results of Goedeker et al. [17].

Nephrotoxicity is a major concern with the use of ASOs $[3,15]$. A significantly increased risk of renal impairment was observed in patients with Duchenne muscular dystrophy treated with drisapersen [36]. In the abovementioned study on inotersen, three cases of glomerulonephritis and one case of tubulointerstitial nephritis were observed in 112 subjects with hereditary transthyretin amyloidosis [4]. In pivotal studies on nusinersen, shifts to high urea nitrogen or creatinine were observed only in individual cases. Additionally, proteinuria did not occur more frequently in nusinersentreated subjects than in control subjects, so the studies did not provide evidence of renal impairment [11, 14, 29]. In the study of Goedeker et al. proteinuria was the most common laboratory abnormality. However, it was never persistent and often attributed to sample contamination [17]. A safety evaluation of 18 adult patients with SMA type 3 and type 4 treated with nusinersen over a 14-month period revealed no evidence of renal dysfunction during therapy [13]. In our cohort, mean creatinine levels at baseline were decreased in both the SMA type 2 and SMA type 3 subgroups, with SMA type 2 patients having lower values than SMA type 3 patients ( 0.31 vs. $0.49 \mathrm{mg} / \mathrm{dL})$. These observations are not surprising since creatinine levels correlate with skeletal muscle mass and are consistent with a study conducted in 238 patients with SMA types $1-3$. In line with our results, the authors found 1.7-fold higher creatinine levels in SMA type 3 than in SMA type 2. Furthermore, serum creatinine correlated with SMA type, $S M N 2$ gene copy number, motor function, and severity of denervation. The authors conclude that creatinine is a candidate biomarker for SMA progression and suggest investigating whether creatinine levels respond to new SMA therapies [2, 22]. Remarkably, only nine SMA type 3 patients from our cohort had normal creatinine values at baseline, and six of them dropped below the LLN during the course of therapy, with one decrease classified as persistent. Furthermore, mean creatinine levels decreased in the SMA type 3 subgroup and the total cohort, possibly indicating further disease progression under therapy. With regard to nephrotoxicity, we found no case of a relevant increase in either serum creatinine or urea nitrogen. However, the use of serum creatinine as a marker of renal function is strongly limited in SMA patients for the reason already mentioned above. Therefore, determination of cystatin $\mathrm{C}$ protein might be a more appropriate method for monitoring renal function in patients with SMA [13, 22].

Hepatotoxicity or an increase in liver enzymes are other potential side effects with the use of ASOs [15]. In clinical trials, mipomersen, an ASO used to treat dyslipidemia, resulted in an increased incidence of elevated ALT above $3 \times \mathrm{ULN}$ and hepatic steatosis compared to placebo [33]. With regard to nusinersen, the NURTURE trial showed stable transaminases in presymptomatic children [12]. In ENDEAR and CHERISH studies, there were some shifts in ALT and AST, with no clear difference from the control groups or reports of liver failures [11, 14, 29]. Similar to the results from pediatric studies, only a few and mostly temporary elevations in liver enzymes were observed in our cohort. However, one patient with SMA type 2 with a normal baseline value developed a discrete but persistent increase in AST, while a single SMA type 2 patient showed a successive increase in GGT. Remarkably, both cases would only fulfill grade 1 according to the NCI-CTCAE, defined as enzyme levels up to $3 \times$ ULN [1]. Regardless of the baseline value, no patient in our study developed elevations in liver 
enzymes above threefold the respective baseline value, and no patient with elevated liver enzymes showed increased bilirubin levels (data not shown). In conclusion, there was no evidence of a clinically relevant increase under treatment with nusinersen.

In terms of detecting potential toxicities of nusinersen therapy in adult patients, this study has some limitations. Importantly, despite the relatively long observation period, rare side effects may not have been captured because of the limited cohort size. In outpatients, coagulation parameters were not usually assessed during therapy. Thus, a large proportion of samples and results were from more severely affected patients, which may result in a bias. The other blood samples from outpatients were usually acquired before the first loading dose and each maintenance dose. Therefore, transient changes during loading dosing in these patients may have been missed. Finally, our definition of the duration of deviations from the normal range is somewhat arbitrary. In particular, "persistent" changes may have returned to normal values after the end of the observation period, and conversely, "transient" shifts may have persisted.

\section{Conclusion}

Our data demonstrate a favorable safety profile of nusinersen therapy in adult SMA patients under longer-term "realworld" conditions, regardless of SMA type. In particular, we found no evidence of clinically relevant platelet declines, coagulopathies, or renal or hepatic organ toxicities, which are common concerns with the use of ASOs. Whether this finding is solely attributable to the intrathecal administration route of nusinersen remains speculative.

Supplementary Information The online version contains supplementary material available at https://doi.org/10.1007/s00415-021-10569-8.

Authors' contributions All authors contributed to the study conception and design. Material preparation, data collection and analysis were performed by BS, MN, KK, SB and TH. The first draft of the manuscript was written by BS and all authors commented on previous versions of the manuscript. All authors read and approved the final manuscript.

Funding Open Access funding enabled and organized by Projekt DEAL. This research received no specific grants from any funding agency in the public, commercial or non-profit sectors.

Data availability The data that support the findings of this study are available on request from the corresponding author. The data are not publicly available due to privacy or ethical restrictions.

\section{Declarations}

Conflicts of interest BS received travel reimbursement and speaker honoraria from Biogen. MN declared no conflicts of interest. KK received travel reimbursement and speaker honoraria from Biogen. SB declared no conflicts of interest. ATo declared no conflicts of interest. ATh received travel reimbursement from CSL Behring. BW declared no conflicts of interest. CD declared no conflicts of interest. CK received honoraria from Biogen and Roche. $\mathrm{TH}$ received honoraria from Novartis, Roche and Biogen and research support from Biogen, Roche and AveXis.

Ethical approval The study was approved by the Ethics Committee of the University Duisburg-Essen, Germany (approval number: 18-8071BO) and was therefore performed in accordance with the ethical standards laid down in the 1964 Declaration of Helsinki and its later amendments.

Informed consent Written informed consent was obtained from every patient included in this study.

Open Access This article is licensed under a Creative Commons Attribution 4.0 International License, which permits use, sharing, adaptation, distribution and reproduction in any medium or format, as long as you give appropriate credit to the original author(s) and the source, provide a link to the Creative Commons licence, and indicate if changes were made. The images or other third party material in this article are included in the article's Creative Commons licence, unless indicated otherwise in a credit line to the material. If material is not included in the article's Creative Commons licence and your intended use is not permitted by statutory regulation or exceeds the permitted use, you will need to obtain permission directly from the copyright holder. To view a copy of this licence, visit http://creativecommons.org/licenses/by/4.0/.

\section{References}

1. (2017) National Institutes of Health-National Cancer Institute. Common Terminology Criteria for Adverse Events (CTCAE) Version 5.0. In:U.S. DEPARTMENT OF HEALTH AND HUMAN SERVICES. https://ctep.cancer.gov/protocoldevelopment/elect ronic_applications/ctc.htm. 2017., p https://ctep.cancer.gov/proto coldevelopment/electronic_applications/ctc.htm

2. Alves CRR, Zhang R, Johnstone AJ, Garner R, Nwe PH, Siranosian JJ, Swoboda KJ (2020) Serum creatinine is a biomarker of progressive denervation in spinal muscular atrophy. Neurology 94:e921-e931

3. Bennett CF, Swayze EE (2010) RNA targeting therapeutics: molecular mechanisms of antisense oligonucleotides as a therapeutic platform. Annu Rev Pharmacol Toxicol 50:259-293

4. Benson MD, Waddington-Cruz M, Berk JL, Polydefkis M, Dyck PJ, Wang AK, Planté-Bordeneuve V, Barroso FA, Merlini G, Obici L, Scheinberg M, Brannagan TH, Litchy WJ, Whelan C, Drachman BM, Adams D, Heitner SB, Conceição I, Schmidt HH, Vita G, Campistol JM, Gamez J, Gorevic PD, Gane E, Shah AM, Solomon SD, Monia BP, Hughes SG, Kwoh TJ, McEvoy BW, Jung SW, Baker BF, Ackermann EJ, Gertz MA, Coelho T (2018) Inotersen treatment for patients with hereditary transthyretin amyloidosis. N Engl J Med 379:22-31

5. Bourque PR, Breiner A, Moher D, Brooks J, Hegen H, Deisenhammer F, McCudden CR (2019) Adult CSF total protein: higher upper reference limits should be considered worldwide. A webbased survey. J Neurol Sci 396:48-51

6. Breiner A, Moher D, Brooks J, Cheng W, Hegen H, Deisenhammer F, McCudden CR, Bourque PR (2019) Adult CSF total protein upper reference limits should be age-partitioned and significantly higher than $0.45 \mathrm{~g} / \mathrm{L}$ : a systematic review. J Neurol 266:616-624 
7. Chan JH, Lim S, Wong WS (2006) Antisense oligonucleotides: from design to therapeutic application. Clin Exp Pharmacol Physiol 33:533-540

8. Crooke ST, Baker BF, Kwoh TJ, Cheng W, Schulz DJ, Xia S, Salgado N, Bui HH, Hart CE, Burel SA, Younis HS, Geary RS, Henry SP, Bhanot S (2016) Integrated safety assessment of 2'-O-methoxyethyl chimeric antisense oligonucleotides in nonhuman primates and healthy human volunteers. Mol Ther 24:1771-1782

9. Crooke ST, Baker BF, Pham NC, Hughes SG, Kwoh TJ, Cai D, Tsimikas S, Geary RS, Bhanot S (2018) The effects of 2'-O-methoxyethyl oligonucleotides on renal function in humans. Nucleic Acid Ther 28:10-22

10. Crooke ST, Baker BF, Witztum JL, Kwoh TJ, Pham NC, Salgado N, McEvoy BW, Cheng W, Hughes SG, Bhanot S, Geary RS (2017) The effects of 2'-O-methoxyethyl containing antisense oligonucleotides on platelets in human clinical trials. Nucleic Acid Ther 27:121-129

11. Darras BT, Farrar MA, Mercuri E, Finkel RS, Foster R, Hughes SG, Bhan I, Farwell W, Gheuens S (2019) An integrated safety analysis of infants and children with symptomatic spinal muscular atrophy (SMA) treated with nusinersen in seven clinical trials. CNS Drugs 33:919-932

12. De Vivo DC, Bertini E, Swoboda KJ, Hwu WL, Crawford TO, Finkel RS, Kirschner J, Kuntz NL, Parsons JA, Ryan MM, Butterfield RJ, Topaloglu H, Ben-Omran T, Sansone VA, Jong YJ, Shu F, Staropoli JF, Kerr D, Sandrock AW, Stebbins C, Petrillo M, Braley G, Johnson K, Foster R, Gheuens S, Bhan I, Reyna SP, Fradette S, Farwell W, Group NS (2019) Nusinersen initiated in infants during the presymptomatic stage of spinal muscular atrophy: Interim efficacy and safety results from the Phase 2 NURTURE study. Neuromuscul Disord 29:842-856

13. De Wel B, Goosens V, Sobota A, Van Camp E, Geukens E, Van Kerschaver G, Jagut M, Claes K, Claeys KG (2020) Nusinersen treatment significantly improves hand grip strength, hand motor function and MRC sum scores in adult patients with spinal muscular atrophy types 3 and 4. J Neurol 268(3):923-935

14. Finkel RS, Mercuri E, Darras BT, Connolly AM, Kuntz NL, Kirschner J, Chiriboga CA, Saito K, Servais L, Tizzano E, Topaloglu H, Tulinius M, Montes J, Glanzman AM, Bishop K, Zhong ZJ, Gheuens S, Bennett CF, Schneider E, Farwell W, De Vivo DC, Group ES (2017) Nusinersen versus sham control in infantileonset spinal muscular atrophy. N Engl J Med 377:1723-1732

15. Frazier KS (2015) Antisense oligonucleotide therapies: the promise and the challenges from a toxicologic pathologist's perspective. Toxicol Pathol 43:78-89

16. Geary RS, Yu RZ, Levin AA (2001) Pharmacokinetics of phosphorothioate antisense oligodeoxynucleotides. Curr Opin Investig Drugs 2:562-573

17. Goedeker NL, Gibbons JL, Varadhachary AS, Connolly AM, Zaidman CM (2021) Laboratory monitoring of nusinersen safety. Muscle Nerve. https://doi.org/10.1002/mus.27217

18. Hagenacker T, Wurster CD, Günther R, Schreiber-Katz O, Osmanovic A, Petri S, Weiler M, Ziegler A, Kuttler J, Koch JC, Schneider I, Wunderlich G, Schloss N, Lehmann HC, Cordts I, Deschauer M, Lingor P, Kamm C, Stolte B, Pietruck L, Totzeck A, Kizina K, Mönninghoff C, von Velsen O, Ose C, Reichmann H, Forsting M, Pechmann A, Kirschner J, Ludolph AC, Hermann A, Kleinschnitz C (2020) Nusinersen in adults with 5q spinal muscular atrophy: a non-interventional, multicentre, observational cohort study. Lancet Neurol 19:317-325

19. Jaffé M (1886) Ueber den Niederschlag, welchen Pikrinsäure in normalem Harn erzeugt und über eine neue Reaction des Kreatinins. Z Physiol Chem 10:391-400

20. Jason TL, Koropatnick J, Berg RW (2004) Toxicology of antisense therapeutics. Toxicol Appl Pharmacol 201:66-83
21. Kessler T, Latzer P, Schmid D, Warnken U, Saffari A, Ziegler A, Kollmer J, Mohlenbruch M, Ulfert C, Herweh C, Wildemann B, Wick W, Weiler M (2020) Cerebrospinal fluid proteomic profiling in nusinersen-treated patients with spinal muscular atrophy. J Neurochem 153:650-661

22. Kim SW, Jung HW, Kim CH, Kim KI, Chin HJ, Lee H (2016) A new equation to estimate muscle mass from creatinine and cystatin C. PLoS ONE 11:e0148495

23. Korte W, Clarke S, Lefkowitz JB (2000) Short activated partial thromboplastin times are related to increased thrombin generation and an increased risk for thromboembolism. Am J Clin Pathol 113:123-127

24. Lefebvre S, Burglen L, Reboullet S, Clermont O, Burlet P, Viollet L, Benichou B, Cruaud C, Millasseau P, Zeviani M et al (1995) Identification and characterization of a spinal muscular atrophydetermining gene. Cell 80:155-165

25. Lefebvre S, Burlet P, Liu Q, Bertrandy S, Clermont O, Munnich A, Dreyfuss G, Melki J (1997) Correlation between severity and SMN protein level in spinal muscular atrophy. Nat Genet 16:265-269

26. Maggi L, Bello L, Bonanno S, Govoni A, Caponnetto C, Passamano L, Grandis M, Trojsi F, Cerri F, Ferraro M, Bozzoni V, Caumo L, Piras R, Tanel R, Saccani E, Meneri M, Vacchiano V, Ricci G, Soraru' G, D’Errico E, Tramacere I, Bortolani S, Pavesi G, Zanin R, Silvestrini M, Politano L, Schenone A, Previtali SC, Berardinelli A, Turri M, Verriello L, Coccia M, Mantegazza R, Liguori R, Filosto M, Marrosu G, Siciliano G, Simone IL, Mongini T, Comi G, Pegoraro E (2020) Nusinersen safety and effects on motor function in adult spinal muscular atrophy type 2 and 3. J Neurol Neurosurg Psychiatry 91:1166-1174

27. McCudden CR, Brooks J, Figurado P, Bourque PR (2017) Cerebrospinal fluid total protein reference intervals derived from 20 years of patient data. Clin Chem 63:1856-1865

28. Mercuri E, Bertini E, Iannaccone ST (2012) Childhood spinal muscular atrophy: controversies and challenges. Lancet Neurol $11: 443-452$

29. Mercuri E, Darras BT, Chiriboga CA, Day JW, Campbell C, Connolly AM, Iannaccone ST, Kirschner J, Kuntz NL, Saito K, Shieh PB, Tulinius M, Mazzone ES, Montes J, Bishop KM, Yang Q, Foster R, Gheuens S, Bennett CF, Farwell W, Schneider E, De Vivo DC, Finkel RS, Group CS (2018) Nusinersen versus sham control in later-onset spinal muscular atrophy. N Engl J Med 378:625-635

30. Mina A, Favaloro EJ, Mohammed S, Koutts J (2010) A laboratory evaluation into the short activated partial thromboplastin time. Blood Coagul Fibrinolysis 21:152-157

31. Munsat TL, Davies KE (1992) International SMA consortium meeting. (26-28 June 1992, Bonn, Germany). Neuromuscul Disord 2:423-428

32. Müschen LH, Osmanovic A, Binz C, Jendretzky KF, Ranxha G, Bronzlik P, Abu-Fares O, Wiehler F, Möhn N, Hümmert MW, Gingele S, Götz F, Stangel M, Skripuletz T, Schreiber-Katz O, Petri S (2021) Cerebrospinal fluid parameters in antisense oligonucleotide-treated adult $5 \mathrm{q}$-spinal muscular atrophy patients. Brain Sci 11(3):296

33. Panta R, Dahal K, Kunwar S (2015) Efficacy and safety of mipomersen in treatment of dyslipidemia: a meta-analysis of randomized controlled trials. J Clin Lipidol 9:217-225

34. Roch-Ramel F (1967) An enzymic and fluorophotometric method for estimating urea concentrations in nanoliter specimens. Anal Biochem 21:372-381

35. Shaw LM, Strømme JH, London JL, Theodorsen L (1983) International Federation of Clinical Chemistry, (IFCC), Scientific Committee, Analytical Section. IFCC methods for the measurement of catalytic concentration of enzymes. Part 4. IFCC method for 
gamma-glutamyltransferase [(gamma-glutamyl)-peptide: amino acid gamma-glutamyltransferase, EC 2.3.2.2]. J Clin Chem Clin Biochem 21:633-646

36. Shimizu-Motohashi Y, Murakami T, Kimura E, Komaki H, Watanabe N (2018) Exon skipping for Duchenne muscular dystrophy: a systematic review and meta-analysis. Orphanet J Rare Dis 13:93

37. Stolte B, Totzeck A, Kizina K, Bolz S, Pietruck L, Mönninghoff C, Guberina N, Oldenburg D, Forsting M, Kleinschnitz C, Hagenacker T (2018) Feasibility and safety of intrathecal treatment with nusinersen in adult patients with spinal muscular atrophy. Ther Adv Neurol Disord 11:1756286418803246

38. Szabó L, Gergely A, Jakus R, Fogarasi A, Grosz Z, Molnár MJ, Andor I, Schulcz O, Goschler Á, Medveczky E, Czövek D, Herczegfalvi Á (2020) Efficacy of nusinersen in type 1, 2 and 3 spinal muscular atrophy: real world data from Hungarian patients. Eur J Paediatr Neurol. https://doi.org/10.1016/j.ejpn.2020.05.002

39. Walter MC, Wenninger S, Thiele S, Stauber J, Hiebeler M, Greckl E, Stahl K, Pechmann A, Lochmuller H, Kirschner J, Schoser B (2019) Safety and treatment effects of nusinersen in longstanding adult 5q-SMA type 3 -a prospective observational study. $\mathrm{J}$ Neuromuscul Dis 6:453-465
40. Witztum JL, Gaudet D, Freedman SD, Alexander VJ, Digenio A, Williams KR, Yang Q, Hughes SG, Geary RS, Arca M, Stroes ESG, Bergeron J, Soran H, Civeira F, Hemphill L, Tsimikas S, Blom DJ, O'Dea L, Bruckert E (2019) Volanesorsen and triglyceride levels in familial chylomicronemia syndrome. N Engl J Med 381:531-542

41. Wurster CD, Koch JC, Cordts I, Dreyhaupt J, Otto M, Uzelac Z, Witzel S, Winter B, Kocak T, Schocke M, Weydt P, Wollinsky K, Ludolph AC, Deschauer M, Lingor P, Tumani H, Hermann A, Gunther R (2019) Routine cerebrospinal fluid (CSF) parameters in patients with spinal muscular atrophy (SMA) treated with nusinersen. Front Neurol 10:1179

42. Wurster CD, Winter B, Wollinsky K, Ludolph AC, Uzelac Z, Witzel S, Schocke M, Schneider R, Kocak T (2019) Intrathecal administration of nusinersen in adolescent and adult SMA type 2 and 3 patients. J Neurol 266:183-194

43. Yeo CJJ, Simeone SD, Townsend EL, Zhang RZ, Swoboda KJ (2020) Prospective cohort study of nusinersen treatment in adults with spinal muscular atrophy. J Neuromuscul Dis 7:257-268 